\title{
Théologiques
}

Revue interdisciplinaire d'études religieuses

\section{Une décentralisation salutaire ou une révision en profondeur du gouvernement de l'Église catholique ?}

\section{Gilles Routhier}

Volume 27, numéro 2, 2019

Une décentralisation salutaire de l’Église catholique?

URI : https://id.erudit.org/iderudit/1069131ar

DOI : https://doi.org/10.7202/1069131ar

Aller au sommaire du numéro

Éditeur(s)

Institut d'études religieuses de l'Université de Montréal

ISSN

1188-7109 (imprimé)

1492-1413 (numérique)

Découvrir la revue

Citer cet article

Routhier, G. (2019). Une décentralisation salutaire ou une révision en profondeur du gouvernement de l'Église catholique ? Théologiques, 27(2), 37-57. https://doi.org/10.7202/1069131ar
Résumé de l'article

On parle abondamment de la « décentralisation » du gouvernement de l’Église catholique. Il y a une admission implicite, dans cette manière de parler, du fait que l'Église de Rome est le centre et que les autres Églises gravitent autour de ce centre. Et si l'on pensait autrement les choses, dans une perspective de communion entre les Églises. Cela conduirait probablement à envisager autrement la réforme des institutions « centrales » de gouvernement de l’Église universelle. Non seulement on revaloriserait les conférences épiscopales ou les assemblées régionales d'évêques, mais on envisagerait réellement une déconcentration des organismes de la curie. Telle est la proposition avancée dans cet article. 


\title{
Une décentralisation salutaire ou une révision en profondeur du gouvernement de l'Église catholique?
}

\author{
Gilles RouthiER" \\ Théologie et de sciences religieuses \\ Université Laval (Québec, Canada)
}

La question de l'exercice du ministère primatial dans l'Église catholique fait l'objet de débat depuis la phase préparatoire du concile Vatican II. Dans ce débat, c'est souvent, bien que non exclusivement, la curie romaine — question que s'était réservée Paul VI — qui est mise en cause. Le concile Vatican II, à la suite d'âpres débats, est parvenu à deux propositions aptes à modifier le gouvernement de l'Église catholique: d'une part, il a élaboré la doctrine de la collégialité des évêques et, d'autre part, sur le plan institutionnel cette fois, il a proposé la création du synode des évêques, organisme créé par Paul VI et qui a évolué avec le temps ${ }^{1}$. Toujours sur le plan institutionnel, Paul VI a procédé à une importante réforme de la curie romaine. Ce travail de réforme de la curie, jamais achevé, s'est poursuivi sous le pontificat de Jean-Paul II qui a vu la publication de la Constitution apostolique Pastor Bonus. Récemment, la question a été reprise par François².

Par ailleurs, depuis Vatican II, les dialogues œcuméniques ont approfondi la question de la primauté. Aujourd'hui, personne ne remet en cause

* Gilles Routhier est doyen et professeur titulaire et à la Faculté de théologie et de sciences religieuses de l'Université Laval. Il y enseigne l'ecclésiologie et la théologie pratique. Ses recherches actuelles portent principalement sur Vatican II, son histoire, son enseignement, son herméneutique et sa réception, ce qui l'a conduit à une lecture de l'évolution de l'Église catholique. Il est l'auteur, avec Joseph Famerée, d'un ouvrage à paraître en 2020 sur la réforme de l’Église, aux Éditions du Cerf (Paris).

1. Sur la création du synode des évêques et son évolution, on verra Routhier (2005; 2006a et 2006b). Sur la réforme récente de l'institution, voir Borras (2019).

2. Sur le débat entourant la réforme de la curie romaine depuis Vatican II jusqu'à François, voir G. Routhier (à paraître en 2020). 
la primauté de l'évêque de Rome. Toutefois, en commençant par Paul VI, les successeurs des apôtres Pierre et Paul ont reconnu que «le pape [...] constitue sans aucun doute l'obstacle le plus grave sur la route de l'œcuménisme» (Paul VI 1967, 498). Depuis, le diagnostic s'est précisé. Ce n'est pas «le pape» en soi qui est un obstacle ou la primauté de l'évêque de Rome, mais une forme d'exercice de la primauté. Faisant écho à la déclaration de Paul VI, Vissert't Hooft déclarait en 1985: "Le vrai problème, c'est la papauté telle qu'elle existe aujourd'hui ${ }^{3}$.» Ce diagnostic a été repris devait être assumé par Jean-Paul II, d'abord lors de la réception du patriarche Dimitrios $1^{\text {er }}$, le 6 décembre 1987. À cette occasion, Jean-Paul II déclarait: "Dans la perspective de cette communion parfaite que nous voulons rétablir, je prie l'Esprit-Saint de nous donner sa lumière et d'éclairer tous les pasteurs et théologiens de nos Églises, afin que nous puissions chercher, évidemment ensemble, les formes dans lesquelles ce ministère pourra réaliser un service d'amour reconnu par les uns et les autres ${ }^{4}$.» Puis, c'est presque pratiquement dans les mêmes termes qu'il s'exprimait dans son encyclique Ut unum sint du 25 mai $1995^{5}$.

Dans son exhortation apostolique Evangelii gaudium, François reprend le flambeau. Citant ce passage de l'encyclique de Jean-Paul II, il appelle à une "conversion de la papauté". Dans son esprit, la conversion pastorale de la papauté inclut «les structures centrales de l'Église universelle [qui] ont besoin d'écouter l'appel à une conversion pastorale» $\left(\mathrm{n}^{\circ} 32\right)$. Le pape fait alors deux propositions: une revalorisation du rôle des conférences épiscopales et une "décentralisation salutaire ${ }^{6}$.

3. Ces propos, prononcés dans le cadre d'une réflexion sur «Magistri et magisterium dans le dialogue œcuménique", sont publiés dans Maury $(2001,178)$.

4. Jean-Paul II, homélie, 6 décembre 1987, La Documentation catholique, 85, no 1954 (17 janvier 1988), p. 88.

5. Voir le n 95 de son encyclique: «J'écoute la requête qui m’est adressée de trouver une forme d'exercice de la primauté ouverte à une situation nouvelle, mais sans renoncement aucun à l'essentiel de sa mission. » Cet appel avait donné lieu à plusieurs publications. Du côté catholique, on verra Il Primato del Successore di Pietro (1998); Quinn (1999); Puglisi (1999); Tihon (2000); Nilson (2001); Pottmeyer (2001).

6. Voir ce qu'il affirmait déjà au n $n^{\circ} 16$ : "Je ne crois pas non plus qu'on doive attendre du magistère papal une parole définitive ou complète sur toutes les questions qui concernent l'Église et le monde. Il n'est pas opportun que le Pape remplace les Épiscopats locaux dans le discernement de toutes les problématiques qui se présentent sur leurs territoires. En ce sens, je sens la nécessité de progresser dans une “décentralisation" salutaire. » Au no 32, il reprenait la question de la centralisation: «Une excessive centralisation, au lieu d'aider, complique la vie de l'Église et sa dynamique missionnaire. » 
J'aborderai dans cet article les deux propositions de François, soit celle de revaloriser les conférences épiscopales, leur conférant une certaine autorité doctrinale, et l'autre, liée à la première, de procéder à une «conversion pastorale » des structures centrales de l'Église, ce qui engage une décentralisation salutaire.

\section{Revaloriser les conférences épiscopales}

Déjà en 1967, à la suite des débats conciliaires, on avait souhaité la mise en œuvre du principe de subsidiarité dans la révision du Code de droit canonique. En pratique, ce principe de philosophie sociale, qui consiste à confier aux groupements d'ordre inférieur les fonctions qu'ils sont en mesure de remplir eux-mêmes plutôt que de les confier à une collectivité plus vaste ou de rang plus élevé, a été bien peu mis en valeur dans la réforme du Codex et encore moins dans les réformes institutionnelles et les pratiques effectives 7 . Le plus grave inconvénient dans l'usage de ce principe formulé dans l'encyclique Quadragesimo anno ${ }^{8}$, c'est qu'il postule qu'il y a des groupements d'ordre inférieur et des collectivités de rang plus élevé. Est-ce bien ainsi que l'on conçoit l'Église? Est-elle constituée à partir de regroupements (dans le cas, les conférences épiscopales nationales ou les regroupements continentaux d'évêques) et d'une collectivité de rang plus élevé?

Au concile Vatican II, on avait évoqué à quelques reprises le principe de subsidiarité au moment du débat sur les conférences épiscopales, lors de la deuxième session. Ainsi, Mgr Gargitter y fit appel dans son plaidoyer en faveur d'une plus large décentralisation de l'Église'. Mgr Schoiswohl y faisait également référence, soulignant que:

1. Ce qui appartient de droit divin à un évêque, dans le gouvernement de son diocèse, devrait être le moins possible limité par l'autorité suprême du souverain pontife.

2. Dans l'administration de l'Église, qui est souvent basée sur des lois ecclésiastiques, le principe suivant devrait être tenu: les diocèses et les évêques devraient avoir toutes les compétences appro-

7. Voir l'article déterminant de Komonchak (1988).

8. Pie XI, Quadragesimo Anno, AAS 23 (1931, 203); La Documentation catholique 1931, col. 1427.

9. AS II/4, p. 454. Le cardinal König souscrivait à cette proposition (p. 479), ainsi que Mgr Baudoux (p. 833-834). 
priées et l'administration centrale ne devrait avoir que ce qui lui est nécessaire.

On imaginait alors le passage d'un régime de concession de facultés aux évêques à un régime de réserve de certaines compétences au Siège apostolique. Dans la foulée de la Constitution sur la liturgie qui reconnaissait, au n $\mathrm{n}^{\circ} 22$, à l'évêque d'un diocèse et aux assemblées d'évêques compétentes sur un territoire donné (territoriales episcoporum coetus) une autorité légitime en matière liturgique, on souhaitait redonner aux évêques dans leur diocèse et aux assemblées épiscopales tous les pouvoirs nécessaires à l'exercice de leur fonction, hormis celles que, pour le bien de l'ensemble, devait se réserver le Siège apostolique. On imaginait le passage d'un régime de concession de facultés à la reconnaissance des droits de l'évêque diocésain et des assemblées d'évêques. L'inversion des logiques — de la concession à la réservation des pouvoirs - semblait être un acquis à la suite du débat sur la charge pastorale des évêques ${ }^{10}$. Au régime de l'indult semblait devoir succéder la reconnaissance du fait qu'une autorité appartient de droit aux diverses assemblées d'évêques compétentes sur un territoire donné plus à même d'opérer les diverses adaptations aux cultures sous l'autorité des conférences épiscopales.

Dans les faits, les compétences attribuées aux conférences épiscopales, en matière liturgique d'abord, ont été de plus en plus réduites. On en vint même à remettre en cause leur mandatum docendi (voir Routhier 2006c; 2010), compris désormais à l'intérieur de règles très strictes ${ }^{11}$. C'est cette autorité doctrinale des conférences épiscopales, amoindries depuis 1985, que François veut rehausser lorsqu'il parle de décentralisation:

10. Voir le discours de Paul VI à la fin de la deuxième période; la lettre apostolique Pastorale munus et $C D$ 8. On s'est un moment demandé s'il s'agissait de facultés "concédées" (voir le résumé italien donné par l'Osservatore romano) ou "accordées » (La Croix) ou des «nombreux pouvoirs que [...] faire partie de la compétence des évêques» (Discours de clôture de Paul VI). Le premier schéma De episcopis parlait de facultés concédées.

11. On verra l'article 1 des normes complémentaires concernant les conférences des évêques dans la Lettre apostolique en forme de motu proprio de Jean-Paul II sur la nature théologique et juridique des conférences épiscopales (Apostolos suos): "Pour que les déclarations doctrinales de la Conférence des Évêques, conformément au n. 22 de la présente Lettre, constituent un magistère authentique et pour qu'elles puissent être publiées au nom de la Conférence elle-même, il est nécessaire qu'elles soient approuvées à l'unanimité des membres Évêques ou bien que, approuvées en réunion plénière au moins par les deux tiers des Prélats appartenant à la Conférence avec voix délibérative, elles obtiennent la reconnaissance (recognitio) du Siège apostolique. » 
Le Concile Vatican II a affirmé que, d'une manière analogue aux antiques Églises patriarcales, les conférences épiscopales peuvent "contribuer de façons multiples et fécondes à ce que le sentiment collégial se réalise concrètement ». Mais ce souhait ne s'est pas pleinement réalisé, parce que n'a pas encore été suffisamment explicité un statut des conférences épiscopales qui les conçoive comme sujet d'attributions concrètes, y compris une certaine autorité doctrinale authentique. Une excessive centralisation, au lieu d'aider, complique la vie de l'Église et sa dynamique missionnaire. (Eg 32)

En fait, on peut se demander s'il s'agit d'une décentralisation, ou s'il ne s'agit pas, plutôt, de reconnaître les compétences propres et qui leur appartiennent aux évêques locaux et aux assemblées d'évêques compétentes sur un territoire donné, comme le fait la Constitution sur la liturgie qui renverse l'approche mise en avant par Mediator Dei qui statuait, au $\mathrm{n}^{\circ} 58$, que « au seul Souverain Pontife appartient le droit de reconnaître et établir tout usage concernant le culte divin [...]; le droit et le devoir des évêques est de veiller diligemment à l'exacte observation des préceptes des saints canons sur le culte divin ${ }^{12}$ ». En fait, il ne s'agit pas de décentraliser, ce qui reviendrait à prétendre que Rome est le centre et qu'il peut concéder certains de ses pouvoirs aux évêques locaux ou aux assemblées légitimes d'évêques, mais de reconnaître que ces pouvoirs appartiennent aux évêques locaux ou réunis en assemblée. C'est en somme renouer, par-delà des siècles, avec ce qui est véritablement traditionnel. Pour mémoire, il suffit de rappeler que, désireux de conclure le concile de Trente qui avait déjà trop duré (1545-1563), les évêques s'étaient volontairement dessaisis de l'autorité dont ils avaient joui jusque-là en confiant au pape le soin de publier le nouveau bréviaire et le nouveau missel $(1563)^{13}$. Il ne faut pas croire cependant qu'il s'agit là d'une pratique ininterrompue. Il faudra attendre 1588, à la suite de la création de la congrégation des rites sacrés, pour que toute initiative en matière liturgique soit retirée aux évêques.

François, à la suite de Paul VI, a commencé à reconnaître les limites d'un discours universel. En effet, François reprend une indication fort peu reçue de Paul VI, suivant laquelle «Face à des situations aussi variées, il nous est difficile de prononcer une parole unique, comme de proposer une solution qui ait une valeur universelle. Telle n'est pas notre ambition, ni même notre mission. Il revient aux communautés chrétiennes d'analyser

12. Pie XII, Encyclique Mediator Dei, AAS, p. 544.

13. Voir Concile de Trente, Session XXV, décret sur l'Index, le catéchisme, le bréviaire et le missel. 
avec objectivité la situation propre de leur pays» (Octogesima adveniens 4, repris dans Eg 184). Paul VI reconnaissait qu'il appartenait aux communautés chrétiennes d'analyser les situations propres de leur pays. François ne fait pas autre chose lorsqu'il déclare que les solutions inculturées, attentives aux traditions et aux défis locaux, doivent être recherchées. Cela témoigne de sa conception de l'exercice du pontificat. Cela se révèle sous sa plume à quelques reprises, d'abord dans Evangelii gaudium lorsqu'il écrit que "Ce n'est pas la tâche du Pape de présenter une analyse détaillée et complète de la réalité contemporaine, mais j'exhorte toutes les communautés à avoir "l'attention constamment éveillée aux signes des temps" " (Eg 51). On retrouve cela à nouveau, en filigrane, dans divers passages d'Amoris laetetia. Déjà au n ${ }^{\circ} 2$, il décourage ceux qui pensent pouvoir "tout résoudre en appliquant des normes générales », comprendre universelles. Enchaînant, il réaffirme que "tous les débats doctrinaux, moraux ou pastoraux ne doivent pas être tranchés par des interventions magistérielles ", comprendre pontificales $\left(\mathrm{n}^{\circ} 3\right)$. Non seulement il recadre les attentes, mais il délimite la fonction magistérielle en requalifiant du même coup la fonction proprement pastorale de discernement, au plus près des situations toujours complexes. Il y revient au terme de son exhortation:

Si l'on tient compte de l'innombrable diversité des situations concrètes, comme celles mentionnées auparavant, on peut comprendre qu'on ne devait pas attendre du Synode ou de cette Exhortation une nouvelle législation générale du genre canonique, applicable à tous les cas. Il faut seulement un nouvel encouragement au discernement responsable personnel et pastoral des cas particuliers. $\left(\mathrm{n}^{\circ} 300\right)$

Il propose donc un exercice de la primauté qui laisse aux acteurs locaux (évêques et assemblées épiscopales) le soin de discerner et de juger. S'il demande fréquemment aux pasteurs de discerner, il renvoie également fréquemment aux regroupements d'évêques, soit les conférences épiscopales ou les assemblées continentales d'évêques. Déjà, dans le premier document important de son pontificat (Evangelii gaudium), François référait à 22 reprises à des documents de conférences épiscopales (des États-Unis, de France, du Brésil, des Philippines, de l'Inde), ou d'assemblées régionales d'évêques (en particulier du CELAM, mais aussi de l'Europe). Il ne s'agissait pas d'un acte fortuit, car il récidivait dans son encyclique Laudato Sì. Il y renvoyait à deux reprises à des regroupements continentaux d'évêques, la FABC et le CELAM, et à des documents des conférences épiscopales de 
l'Afrique du Sud, de l'Allemagne, de l'Argentine, de l'Australie, du Brésil, de la Bolivie, du Canada, des États-Unis, du Japon, de la Nouvelle-Zélande, du Mexique, du Paraguay, des Philippines, du Portugal, de la République dominicaine. On n'est donc pas étonné qu'il poursuive dans la même veine dans Amoris laetetia. Cette fois, on recense neuf références à des documents des conférences épiscopales nationales (Espagne, Corée, Argentine, Mexique, Colombie, Chili, Australie, Italie et Kenya) et une à la Conférence générale de l'épiscopat latino-américain d'Aparecida. Il assume donc le magistère des conférences épiscopales ou des regroupements continentaux d'évêques. Ainsi, sans modifier (encore) les règles régissant l'exercice du magistère des conférences épiscopales établies dans le Motu proprio "Apostolos Suos", François revalorise le magistère des conférences épiscopales, ne se contentant pas d'observer, comme il le faisait dans Evangelii gaudium, "que n'a pas encore été suffisamment explicité un statut des conférences épiscopales qui les conçoive comme sujet d'attributions concrètes, y compris une certaine autorité doctrinale authentique » (Eg 32). Il offre, de manière très concrète, une réalisation de ce vœu.

En somme, s'agit-il d'en appeler à une décentralisation salutaire ou à l'application du principe de subsidiarité ou ne s'agirait-il pas, plus simplement, de reconnaître aux évêques et aux assemblées d'évêques légitimes, des compétences qui leur appartiennent, mais qu'on limite fréquemment en concentrant entre les mains du pontife romain et de sa curie la détermination de l'ensemble des règles qui orienteront la conduite des Églises dont les évêques sont chargés à titre de vicaires du Christ ? Certes, la communion de toutes les Églises requiert que des mécanismes de concertation entre les Églises contribuent à nourrir et à protéger l'unité de l'Église tout entière. Mais le respect des compétences des évêques locaux ne doit pas conduire au désordre et à l'anarchie. De plus, on peut très bien recourir à la réserve de certaines questions au Siège apostolique ${ }^{14}$.

\section{La «conversion pastorale» des «structures centrales de l'Église universelle»}

On s'entend, d'une part, sur l'objectif à poursuivre: la conversion pastorale. Tout doit être pensé en fonction de cet objectif. Cela s'applique naturellement également à la revalorisation des conférences épiscopales dont nous avons parlé plus haut. En somme, un changement de mode dans le

14. Sur le rapport entre unité et diversité, voir Lanne (1997; 1969 et 1987). 
gouvernement de l'Église catholique est finalisé par la poursuite de la fin pastorale, ce qui signifie que c'est l'inculturation de l'Évangile dans tous les espaces humains qui commande toute réforme.

Ceci dit, nous l'avons déjà noté au passage, il est préférable le mieux est de réfléchir à partir d'un cadre ecclésiologique fondé sur la communion des Églises, assurant la place qui revient à l'Église de Rome ( «sans préjudice du primat de la Chaire de Pierre qui préside au rassemblement universel de la charité » - $L g$ 13), que de prendre les choses à partir d'un modèle d'Église universelle, concept qui, au demeurant, est polysémique ${ }^{15}$. Le choix d'un cadre ecclésiologique n'est pas insignifiant (Congar 1962 et Komonchak 1981). Si l'on conçoit l'Église entière comme communion des Églises inscrites dans les diverses cultures du monde, divers processus protègent et nourrissent l'unité (voir Routhier 1994). De plus, la communion est assurée au moyen des assemblées d'évêques, en particulier les synodes régionaux (conciles provinciaux) et généraux (voir de Vries 1974). Ce n'est qu'à partir du moment où l'on conçoit l'Église à partir d'un schème universaliste que se développent les organismes centraux de gouvernement de l'Église, l'institution du collège des cardinaux, sénat du pape (pars corporis papae), des consistoires, qui va nous conduire à l'établissement de la curie romaine. "À la faveur de la Contre-Réforme, la papauté se libéra de la puissance du Sacré Collège, réduisit sa signification théologique et circonscrivit son importance administrative, sans la diminuer, en substituant aux consistoires le système des congrégations cardinalices comme structure fondamentale de la curie romaine.» (Legrand 2000, 145)

Le projet actuel de réforme de la curie, inspiré par un véritable souci missionnaire, veut faire de celle-ci un instrument plus souple au service des Églises locales. C'est ce que laissait entendre un membre du C-9, le cardinal O. Gracias. Celui-ci mettait en valeur le titre provisoire de la future constitution apostolique sur la réforme de la curie, Praedicate Evangelium. De plus, le cardinal «has suggested that the pontiff reorganize the Vatican to make a "decisive shift" toward putting the city-state at the service of local Catholic dioceses around the world, said one of the council's nine members " (McElwee 2018). Cette proposition suggère deux modifications dans les finalités de la curie romaine: le service des Églises locales et l'orienta-

15. On sait que, en français, l'expression «Église universelle» traduit deux expressions latines dont le sens est différent: "Ecclesia universalis" et "Ecclesia universa". Le Code de droit canonique n'utilise que cette dernière expression, ignorant totalement la première. Souvent, en raison de cette polysémie, l'emploi de l'expression «Église universelle» est source de grande confusion. Voir Pagé (1986). 
tion missionnaire. Ceci dit, on pense toujours les choses à partir d'un modèle universaliste, même s'il est tempéré.

Comme le souligne Pottmeyer, l'Église catholique ne peut pas biffer d'un trait un millénaire de son histoire et oblitérer le paradigme universaliste dont elle s'est nourrie pendant des siècles pour revenir simplement à un modèle de communion des Églises ${ }^{16}$. Il s'agit, selon lui, d'harmoniser les deux ecclésiologies, car «la juxtaposition sans médiation des deux ecclésiologies ayant des tendances contraires est la cause de nombreux conflits que nous observons aujourd'hui dans l'Église» (Pottmeyer 1981, 92). Il revient sur cette idée en affirmant que "Si l'Église ne doit pas se briser à cause de l'opposition des deux réactions, il se pose nécessairement la tâche de trouver une médiation, qui fasse dépasser la juxtaposition des deux ecclésiologies et rassemble les éléments de vérité contenus dans chacune - une tâche qui demande pareillement des efforts théoriques et pratiques » (Pottmeyer 1981, 92-93).

Cette dernière affirmation oriente notre tâche. Il ne s'agit pas simplement d'opérer une "conversion pastorale» des "structures centrales de l'Église universelle ». Il s'agit de repenser l'ensemble de la gouvernance de l'Église de manière à faire droit aux éléments de vérité des deux paradigmes ecclésiologiques: une ecclésiologie de communion, ancienne et traditionnelle et remise à jour par Vatican II, et une ecclésiologie universaliste qui a mis en valeur la primauté. L'ecclésiologie de communion a conduit à la revalorisation des conférences épiscopales et du synode des évêques, bien que la création de ces deux organismes ne soit pas encore bien intégrée dans les éléments développés par l'ecclésiologie universaliste. Comme l'affirme Pottmeyer, on est en présence d'une "juxtaposition de fait inéclaircie de structures et d'institutions ecclésiales centrales» (Pottmeyer 1981, 93). On a créé un synode d'évêques, mal arrimé aux autres organismes centraux de gouvernement, telle la curie. On a favorisé l'établissement des conférences épiscopales, mais leurs compétences (munus sanctificandi et mandatum docendi) ont sans cesse été réduites au profit de la curie romaine. Il ne s'agit pas d'abolir l'un ou l'autre des éléments, mais de clarifier leurs compétences respectives. C'est là la tâche de la réforme actuelle. Pas de réforme de la curie sans revalorisation des conférences épiscopales et sans un travail sur les articulations entre celle-ci et celles-là. Comme l'écrit Pottmeyer, « la tâche présente consiste à intégrer

16. «L'Église catholique ne peut se contenter de supprimer une phase de son évolution » (Pottmeyer 1985, 52). Voir aussi Pottmeyer (1981). 
ce qui, dans la théologie pré-conciliaire, est obligatoire, au nouvel acquis d'une ecclésiologie de communio" (Pottmeyer 1985, 52). Nous sommes donc conviés à un approfondissement des deux modèles afin de parvenir à une synthèse.

C'est en gardant à l'esprit ce besoin de synthèse et d'intégration que nous devons travailler à la «conversion pastorale» de la curie comme organisme central de l'Église universelle, nous souvenant qu'il faut, pour y arriver, mieux articuler curie, conférences épiscopales (ou assemblées régionales d'évêques) et synode des évêques. Ayant déjà eu l'occasion de travailler ces diverses questions, je me limiterai, pour la curie, à faire deux propositions ou, mieux, à remettre en avant deux propositions déjà connues.

\section{Des propositions pour nous aider à repenser le gouvernement de l'Église catholique}

Jusqu'ici, les réformes successives de la curie romaine et, plus généralement, du gouvernement central de l'Église catholique ont tenté d'améliorer le système en place, en y apportant quelques correctifs ou encore en ajoutant des organismes à ceux déjà en place. Ainsi, au collège des cardinaux responsables de conseiller le pape, on ajoute le synode des évêques en 1965 et, comme si cela n'était pas assez, le pape François ajoute à ces deux organismes un conseil de neuf cardinaux, créé le 13 avril 2013. Plutôt que d'abolir une institution qui correspondait à la situation de l'Église ou du monde, on ajoute de nouvelles institutions. Il en va ainsi de l'organisme chargé de conseiller le pape: au consistoire, on ajoute deux nouvelles instances dont les attributions entrent en conflit avec l'institution existante. Le gouvernement s'en trouve de ce fait plus dysfonctionnel. Je mettrai donc en avant deux propositions qui peuvent nous aider à penser les choses autrement plutôt que de simplement rapiécer le système que l'on connaît.

Je tiens la première, que je ne présenterai que succinctement, de Michel Camdessus, ancien directeur général du Fonds monétaire international et ancien membre du Conseil pontifical Justice et Paix. Je la relève parce que, précisément, il veut rétablir un équilibre, sans pour autant remettre en cause la primauté (Camdessus, Albert et Boissonnat 2001). Il propose que l'Église adopte un fonctionnement proche de celui en vigueur à la Banque mondiale ou au FMI et qui, à son avis, se rapproche du synode patriarcal. Il y aurait, à Rome, des représentants permanents des épiscopats qui serviraient de relais entre le primat et les patriarches et les présidents des 
assemblées continentales (ou nationales) d'évêques. On sait que cette proposition a été faite à quelques reprises par Mgr Hermaniuk et par le patriarche Maximos IV lors du concile ${ }^{17}$. Dans cette perspective, le siège apostolique confierait aux assemblées d'évêques légitimes le règlement de certaines questions. Cette action se ferait, naturellement, de manière coordonnée, les diverses assemblées gardant un lien étroit avec Rome et l'unité entre elles. Dans cette perspective, la curie romaine trouverait une nouvelle place, plus modeste, certes, mais toujours en lien avec les assemblées d'évêques des divers continents ou nations.

La seconde, que j'ai déjà mise en avant, consiste en une déconcentration - plutôt qu'une décentralisation — de la curie romaine. Si la décentralisation consiste à remettre à des organismes locaux (évêques résidentiels) ou régionaux (conférences épiscopales) des pouvoirs jusque-là exercés par les organes centraux du pouvoir, la déconcentration, en revanche, consiste à répartir sur le territoire les organes d'exercice du pouvoir. Si les grands organismes internationaux nous offrent des exemples d'un tel modèle ${ }^{18}$, on peut trouver également des exemples d'un tel fonctionnement dans l'Église catholique. On en connaît au moins trois cas, tous réalisés au cours du pontificat de Paul VI: le cardinal König préside le Secrétariat pour les noncroyants (1965-1980) tout en demeurant évêque de Vienne, le cardinal Roy est président de la Commission Justice et Paix (1967-1976), du Conseil pour les laïcs (1967-1976) et du Comité pour la famille (19731976) tout en demeurant archevêque de Québec, et le cardinal Willebrands occupe simultanément les fonctions de président du Secrétariat pour l'unité des chrétiens et d'archevêque d'Utrecht (1975-1983). Dans tous ces cas de cardinaux placés à la tête d'organismes de la Curie romaine demeurent des évêques résidentiels et exercent des fonctions épiscopales dans leur diocèse. Ces expériences de déconcentration de la Curie concernent toutes de nouveaux organismes curiaux, et elles ont également en commun le fait de se développer au cours du pontificat de Paul VI et de prendre fin au début du pontificat de Jean-Paul II ${ }^{19}$.

Cette situation inusitée crée un nouveau rapport entre la Curie romaine et les Églises locales. Elle suscite également un modus operandi différent

17. Voir AS II/4, p. 516-519.

18. Par exemple, l'ONU a dispersé à travers le monde le siège de divers organismes qui lui sont rattachés: la FAO à Rome, l'UNESCO à Paris, OACI à Montréal, AIEA à Vienne, OMT à Genève, etc.

19. C'est encore le cas, aujourd'hui, pour la Caritas internationalis présidée par un évêque résidentiel. 
des autres dicastères de la Curie et modifie la composition de ce dicastère, sans compter qu'il répartit autrement le leadership. Le fonctionnement du dicastère devient plus décentralisé et les activités prennent une forme nouvelle (congrès, colloques, etc.), se déroulant la plupart du temps loin de Rome $^{20}$. Ces organismes intègrent un grand nombre de laïcs (hommes et femmes) et réalisent véritablement une collaboration entre évêques, prêtres et laïcs. La composition même de la commission Justice et Paix en est un exemple puisqu'elle fait une large place à des experts qui ne sont pas, par leur culture et leur statut, accoutumés aux appareils romains et à la culture cléricale. Ainsi, au moment de sa création, on trouve parmi les membres de la Commission trois ecclésiastiques et dix laïs, dont deux femmes ${ }^{21}$. De plus, les lieux à partir desquels les choses se pensent sont éclatés et diversifiés: Londres, Québec, Chicago, Rome, etc.

Les activités de la Commission, orientées vers l'étude et l'éducation en vue de l'action, se démarquent par rapport à celles des autres dicastères de la Curie. Cela conduit à l'organisation de grands événements: colloques et congrès. Dès le départ, cette action est conduite en relation étroite avec des partenaires. D'une part, la Commission veut s'appuyer sur les conférences épiscopales, jouant ainsi à fond la carte de la collégialité et de la subsidiarité suivant les orientations du concile. D'autre part, elle souhaite une collaboration soutenue avec le Conseil œcuménique des Églises. Enfin, elle favorise de nombreuses collaborations. Ce pari de la collaboration avec les rassemblements nationaux ou régionaux d'évêques et ce parti pris pour l'œcuménisme se dégagent clairement de ses orientations publiées au terme de la première assemblée plénière de la Commission Justice et Paix, en avril 1967:

Moyens d'action

\section{Avec les catholiques}

La Commission travaillera à travers les organisations et les programmes qui existent, mais quand il le faudra, elle stimulera et au besoin aidera à créer de nouvelles initiatives qui correspondent à des situations nouvelles.

20. Outre les réunions plénières et les assemblées générales tenues à Rome, la Commission organise des réunions régionales, à Boston, en janvier 1967, à Porto Rico, en avril 1967, à New York, en novembre 1967, à Bruxelles, en décembre 1967 et à Beyrouth en avril 1968.

21. La Documentation catholique [DC], T. 64, 1487, col. 197-198. 
La Commission suggère donc:

a) Que chaque Conférence épiscopale, régionale ou nationale [...] étudie la création d'une structure faite de clercs et de laïcs, s'appelant éventuellement «Justice et Paix», qui coopérerait étroitement avec la Commission;

b) Qu'une étroite collaboration soit établie avec le Secrétariat pour l'Unité des chrétiens, et avec les Secrétariats pour les non-croyants et pour les religions non chrétiennes;

c) Qu'on cherche à établir des liens étroits et des modalités de travail communes avec le Conseil des laïs;

d) Que tous les Instituts religieux, particulièrement les Instituts missionnaires, soient activement associés au travail de la Commission;

e) Que la Commission s'assure l'aide des Instituts de recherche, des Universités et d'autres Centres d'enseignement;

f) Qu'un accent tout spécial soit mis sur le rôle des jeunes: et que la Commission s'attache spécialement à stimuler et à inspirer toutes les organisations de jeunesse qui existent au sein de l'Église.

\section{Avec nos frères chrétiens}

La Commission s'efforcera de développer le plus possible les étroites relations déjà amorcées entre son Secrétariat et les organes du Conseil œcuménique des Églises, et de promouvoir une action œcuménique avec tous les organismes chrétiens.

\section{Avec les religions non chrétiennes}

La Commission s'efforcera d'établir une collaboration très étroite avec les religions non chrétiennes.

\section{Avec les organisations séculières}

Sur ce point, la politique de la Commission sera celle de «porte ouverte» à tous les groupes et organismes, gouvernementaux et non gouvernementaux, travaillant dans le domaine du développement: elle accueillera volontiers et diffusera le matériel intéressant qu'elle reçoit, et mettra à la disposition des autres organisations ce que l'Église a à offrir dans ce domaine. Elle s'attachera particulièrement à établir des rapports étroits avec les agences internationales. En tout ceci, elle passera par les voies ordinaires, c'est-à-dire la Secrétairerie d'État, les observateurs du Saint-Siège auprès des Nations Unies et de ses organisations affiliées ${ }^{22}$.

Ce modus operandi diffère beaucoup de celui que l'on retrouve dans les autres dicastères. On a affaire à une activité multipolaire plutôt qu'à

22. Voir la déclaration faite à la suite de la première réunion plénière de la Commission, dans DC, T. 64, 1495, col. 1011-1016. 
une action centralisée, une action qui se développe dans les périphéries, plutôt que de se limiter au centre romain. Gremillon, premier secrétaire de Justice et Paix, décrit son activité au cours de la première année: «Lobbying of various religious orders and national and regional bishops' conferences to establish a Catholic connection of Justpax-like organizations began even before Justpax was inaugurated and continued full-force for the early years of the organization ${ }^{23}$." Tout de suite, on voit comment peuvent être articulées autrement les relations entre un organisme du Saint-Siège et les assemblées régionales ou nationales d'évêques.

La création de nombreuses commissions nationales Justice et Paix, le plus souvent par les conférences épiscopales ${ }^{24}$, et la mise sur pied de SODAPAX (Comité sur la Société, le développement et la paix ${ }^{25}$ ) dès la fin de 1967 sont l'expression de ce travail réalisé en collaboration avec les autres. SODAPAX organise la conférence œcuménique de Beyrouth sur le développement en mars 1968, au cours de laquelle des spécialistes du développement économique rencontrent des responsables des Églises catholiques, protestantes et orthodoxes ${ }^{26}$.

Pour désigner ce travail en réseau et multicentrique, Pelzel parle d'une

chain of auxiliary justice and peace commissions [...] established throughout the various levels of the Catholic Church. These Catholic auxiliary organizations were key parts of Gremillion's vision of a "Christian circulatory system » for justice and peace education, research, and action throughout the world ${ }^{27}$.

Toujours selon Pelzel, «The early days of Justpax combined a flurry of activity to get both the Catholic and ecumenical circulatory systems inaugurated ${ }^{28}{ }$. Suivant le rapport d'activités donné lors de la deuxième

23. J. Gremillion, entrevue avec P. Pelzel. Pamela Pelzel avait amorcé une thèse sur la Commission Justice et Paix. N'achevant pas sa thèse, elle m'a communiqué son matériel. Je la remercie.

24. Dans son rapport présenté aux présidents des conférences épiscopales à l'occasion de l'Assemblée extraordinaire du Synode des évêques (Rome, 1969), le cardinal Roy fait état de la création d'une quarantaine de commissions nationales. DC (1969), T. 66, 1953, p. 1110-1112.

25. Il s'agit d'un organisme conjoint, créé par l'Église catholique et le Conseil œecuménique des Églises.

26. En plus des nombreuses sources imprimées, on trouve un dossier sur la conférence de Beyrouth dans les archives Roy (AAQ 31-21 A, T. 23-5).

27. Voir la correspondance entre Gremillion, Ward, et Norris, Archives Gremillion, Université Notre-Dame [NDUA).

28. Documents in Archives Gremillion, NDUA. 
assemblée générale, en octobre 1967, «in less than half a year, Justpax staff had visited over thirty countries and been part of four Holy See delegations to UN agencies while working on establishing many national and regional Justpax organizations ". L'expression qui décrit ce nouveau modus operandi et que l'on trouve dans les documents de la Commission est «circulatory system». On parlerait aujourd'hui d'un fonctionnement en réseau, la Commission n'ayant pas à tout faire elle-même, se limitant à mettre en mouvement des idées et à mobiliser des forces déjà existantes.

Ainsi, en 1969, en présence des présidents des conférences épiscopales réunis à Rome pour une assemblée extraordinaire du Synode des évêques, le cardinal Roy dresse un bilan de ces collaborations multilatérales, les commissions nationales et SOPDEAX (organisme créé en partenariat avec le Conseil œcuménique des Églises), soulignant particulièrement des rencontres régionales organisées à Niamey, en Amérique latine, à Tokyo, en Allemagne et à Genève ${ }^{29}$. La Commission met aussi sur pied des tables rondes dans différentes régions du monde où se retrouvent les acteurs les plus importants afin d'harmoniser l'aide (financière, matérielle et en personnel) au développement.

Enfin, les documents produits par la commission Justice et Paix sont d'un type particulier. Il s'agit d'études, de réflexions, de propositions, de débats entre experts et pasteurs, etc. Dans le rapport de 1969, le cardinal Roy fait état de cinq études en chantier: sur la seconde décennie du développement des Nations Unies; sur la croissance démographique et le développement; sur la pauvreté dans le monde et l'usage des biens dans l'Église; sur la qualité de vie dans une société technologiquement avancée et la dernière sur l'objection de conscience au service militaire ${ }^{30}$.

L'étude de la Commission Justice et Paix, au cours de ses premières années d'activité, doit donc être située dans le projet de renouveau de la Curie souhaité par Paul VI avec qui le cardinal Roy entretenait des liens très étroits. Paul VI devait du reste lui adresser une lettre apostolique, Octogesima adveniens, à l'occasion du $80^{\mathrm{e}}$ anniversaire de l'encyclique Rerum novarum. Le cardinal Roy participa à toutes les assemblées du Synode des évêques et, à titre de président de Justice et Paix, il fit une contribution spéciale à l'Assemblée synodale de 1971 dont l'un des thèmes

29. Voir «Le travail de la Commission Pontificale "Justice et Paix". Communication aux présidents des Conférences épiscopales présents à Rome pour le Synode extraordinaire des évêques", dans DC (1969), T. 66, 1953, p. 1110-1112.

30. DC (1969), T. 66, 1953, p. 1110-1112. 
principaux était la justice et la paix. À ma connaissance, c'est la seule fois, dans l'histoire de l'Église postconciliaire, que le document final de cette partie de la rencontre synodale fut rédigé par une femme, Barbara Ward ${ }^{31}$.

Nous ne sommes donc pas dépourvus d'expérience en matière de renouveau ou de réforme de la curie. On devrait aujourd'hui construire à partir de l'expérience plutôt que de vivre dans l'amnésie. Si l'on voulait aujourd'hui emprunter cette voie de la déconcentration de la curie, il s'agirait de garder à Rome quelques dicastères (Congrégation pour la doctrine de la foi et des évêques, la Secrétairerie d'État et le Conseil pontifical pour l'unité des chrétiens) ou services (les finances, les communications, etc.) et disperser les autres dicastères à travers le monde. On pourrait, par exemple, localiser la Congrégation pour l'évangélisation des peuples (avec l'université qui en dépend) en Afrique, le dicastère pour le développement intégral en Amérique latine, l'éducation catholique aux États-Unis, le conseil pontifical pour le dialogue interreligieux en Inde, les Églises orientales au Liban, etc. Si l'on ne prend pas une telle mesure, le microcosme romain se reconstituera aussitôt et la curie réformée retombera dans ses ornières. À nouveau, on vivra dans une bulle, loin du monde réel, ce que dénonçait déjà Congar lors de ses voyages à Rome au cours des années 1940: "Mais tout ce luxe, ce marbre, ces palais, ces trésors accumulés, tout cela ne tientil pas les organes centraux de l'Église loin du peuple et de la vie, loin du mouvement réel des choses et des problèmes du monde? Tout cela ne fait-il pas habiter les organes centraux et directeurs de l'Église dans un monde irréel, artificiel caractérisé par une trompeuse immutabilité et un faux air de gloire? »(Congar 2000, 19) 32 Ce monde irréel, détaché des problèmes

31. Barbara Ward, aussi connue sous le nom de Lady Jackson, a été rédactrice du prestigieux hebdomadaire londonien The Economist. Conférencière recherchée, elle publie de nombreux articles et ouvrages et influence plusieurs leaders politiques aux États-Unis, en Afrique, en Inde et en Grande-Bretagne. Elle milite pour le partage des richesses entre les pays et, dès le début du concile, elle s'engage en ce sens. Elle est la première femme à s'exprimer lors d'une Assemble du Synode des évêques (1971).

32. Il réfléchit d'ailleurs à partir des mêmes catégories lors d'un séjour à Rome en 1954 : «[...] le sentiment que j'ai qu'il y a d'un côté Rome, déroulant ses rites à l'intérieur d'un système sans cesse justifié et glorifié [...] et, d'un autre côté, les hommes, leurs questions, leurs attentes. Les deux ne se rencontrent pas.» (p. 296) «Je suis frappé partout de cet irréalisme d'un système qui a ses thèses et ses rites, ses serviteurs aussi, et qui chante sa chanson sans regarder les choses et les problèmes tels qu'ils sont. Le système est satisfait de ses propres affirmations et de ses propres célébrations. Cela se déroule à un autre plan que celui des problèmes réels, dans un autre univers que celui des hommes. » (p. 295) "Y a-t-il, ici, un sens tragique des choses, un sentiment 
concrets, n'est pas propre à l'Église. C'est le problème de toutes les grandes administrations. Cependant, l'Église ne peut pas y demeurer indifférente et concentrer toujours davantage son administration centrale.

\section{Conclusion}

On l'a vu plus haut, il faut privilégier une approche systémique si l'on veut parvenir à une véritable réforme du gouvernement de l'Église catholique. Il serait insuffisant de se fixer sur les seuls organismes centraux de l'Église universelle. Une véritable réforme de la curie engage le développement de la synodalité de toute l'Église et une mise en œuvre plus résolue de la collégialité épiscopale qui trouve une forme d'expression dans les rassemblements légitimes d'évêques sur le plan continental et national. Il est fallacieux de prétendre que ces assemblées ne favorisent qu'une collégialité affective et jamais effective ${ }^{33}$. Non seulement cette distinction n'appartient pas au concile Vatican II, mais sa proposition, à partir de 1985, a pour fonction de dévaloriser le statut et les compétences des conférences épiscopales.

Il ne s'agit pas simplement de réformer la curie, comme si elle n'était pas elle-même une pièce d'un ensemble dont le fonctionnement est inspiré du modèle monarchique plutôt que du modèle synodal. Dans son développement, elle s'insère dans une ecclésiologie universaliste plutôt que dans une ecclésiologie fondée sur la communion des Églises. La réforme de la curie doit s'accompagner d'une revalorisation de l'épiscopat, car les évêques, eux aussi inscrits dans une Église vivant de l'esprit synodal, doivent être redevables. Ainsi, un évêque n'est pas un individu isolé ou détaché de son Église (principe synodal) et séparé de ses frères évêques (principe collégial); de même, son Église n'est pas une île, vivant en autarcie, sans communion effective avec les autres Églises, à commencer par les Églises de sa province et de son pays (principe conciliaire), communion qui s'exprime à travers des assemblées d'évêques au niveau national ou conti-

de leur urgence? Peut-on prendre la longueur d'onde des hommes et suivre le rythme de leurs questions?» (p. 294).

33. La distinction fallacieuse entre affectus et effectus collegialis n'existe pas dans les textes de Vatican II. Pour une étude en profondeur de l'apparition et de la diffusion de cette distinction depuis l'Assemblée extraordinaire du Synode de 1985, voir l'étude déterminante de Winterkamp (2003). Vittorio Peri, en marge du symposium de l'Istituto Paolo VI, Paolo VI e la collegialità, remarquait, à la suite d'une intervention de Komonchak, qu'en latin classique, "affectus" signifie la conscience d'appartenir ou de constituer un collège. Voir Istituto Paolo VI (1995, 67-69 et 279-281). 
nental. Cette inscription d'un évêque dans un collège se traduit également par sa communion avec l'évêque de Rome.

En somme, une véritable réforme de la curie ne doit pas isoler la curie des autres composantes du gouvernement de l'Église catholique. Seules une vision systémique et une appréhension globale de ce gouvernement pourront conduire à une réforme féconde. Enfin, il faut rappeler qu'une telle réforme est finalisée par l'évangélisation ou par le caractère missionnaire de l'Église, ce qui implique qu'elle n'est pas simplement de nature administrative, mais engage une "conversion missionnaire».

\section{Références}

Borras, A. (2019), «Episcopalis communio, mérites et limites d'une réforme institutionnelle ", Nouvelle revue théologique, 141/1, p. 66-88.

Camdessus, M., M. Albert et J. Boissonnat (2001), Notre foi dans le siècle, Paris, Arléa.

Collectif (1998), Il Primato del Successore di Pietro, Cité du Vatican, Libreria Editrice Vaticana.

Congar, Y. (1962), "De la communion des Églises à l'ecclésiologie de l'Église universelle », dans Y. Congar et B. DupuY, dir., L'Épiscopat et l'Église universelle, Paris, Cerf (Unam Sanctam 39).

(2000), Journal d'un théologien (1946-1956), présenté et annoté par E. Fouilloux, avec la collaboration de D. Congar, A. Duval et B. Montagnes, Paris, Cerf.

Istituto Paolo VI (1995), Paolo VI e la collegialità episcopale, Brescia.

Komonchaк, J. A. (1981), «L'Église universelle, communion d'Églises locales », Concilium, 166, p. 56-63

(1988), «Le principe de subsidiarité et sa pertinence ecclésiologique », dans H. Legrand, J. Manzanares et A. Gardía y García, Les conférences épiscopales. Théologie, statut canonique, avenir, Paris, Cerf (Cogitatio fidei 149), p. 391-447.

Lanne, E. (1969), «Pluralisme et unité. Possibilité d'une diversité de typologies dans une même adhésion ecclésiale ", Istina, 15, p. 171-190.

(1997), «Les différences compatibles avec l'unité dans la tradition de l'Église ancienne », dans Tradition et communion des Églises, Leuven, Leuven University Press, p. 359-385.

(1987), «Unité et diversité. Au sujet de quelques propositions récentes ", Irénikon, 60/1, p. 16-46. 
Legrand, H. (2000), "Cardinaux, collège des ", dans Dictionnaire de l'Histoire du christianisme, Paris, Encyclopædia universalis / Albin Michel, p. 145.

Maury, J. (2001), dir., W.A. Vissert't Hooft, pionnier de l'œecuménisme Genève-Rome, Paris, Cerf.

McElwee, J. J. (2018), «Vatican Reorganization Would Be "Decisive Shift," says Cardinal Gracias. New Document Outlining Vatican Bureaucracy Said to Call for Service to Local Churches ", National Catholic Reporter, 30 octobre, disponible sur <www.ncronline.org/news/vatican/vaticanreorganization-would-be-decisive-shift-says-cardinal-gracias $>$.

Nilson, J. (2001), Church Unity and the Papal Office, Grand Rapids, Eerdmans, 2001.

PAGÉ, R. (1986), "Note sur la terminologie employée par le Code de droit canonique de 1983 pour parler de l'Église", dans Collectif, Le nouveau Code de droit canonique, dans Collectif, The New Code of Canon Law, Ottawa, p. 271-274.

PAul VI (1967), «Allocution au Secrétariat pour l'Unité, 28 avril 1967 », Acta Apostolicae Sedis 59.

Pottmeyer, H. J. (1981), "Continuité et innovation dans l'ecclésiologie de Vatican II », dans G. Alberigo, dir., Les Églises après Vatican II. Dynamisme et prospective, Paris, Beauchesne (Théologie historique 61), p. 91-116.

(1985), "Vers une nouvelle phase de réception de Vatican II. Vingt ans d'herméneutique du Concile ", dans G. Alberigo et J.-P. Jossua, dir., La réception de Vatican II, Paris, Cerf (Cogitatio fidei 134), p. 43-64.

(2001) [anglais 1998], Le rôle de la papauté au troisième millénaire. Une relecture de Vatican I et de Vatican II, Paris, Cerf.

Puglisi, J. F. (1999), dir., Petrine Ministry and the Unity of the Church, Collegeville, Liturgical Press, 1999.

Quinn, J. R. (1999), The Reform of the Papacy. The Costly Call to Christian Unity, New York, Herder \& Herder.

Routhier, G. (1994), Le défi de la communion. Une relecture de Vatican II, Montréal et Paris, Médiaspaul (Brèches théologiques 18), p. 121-154. (2005), «Mener à terme l'œuvre amorcée. L'éprouvante expérience de la quatrième session", dans G. Alberigo, dir., Histoire de Vatican II, vol. V., Un concile de transition. La quatrième session et la fin du concile (sept. 65 - déc. 65), Paris, Cerf. 
(2006a), «Le synode», dans G. Routhier et L. Villemin, dir., Nouveaux apprentissages pour l'Église. Mélanges offerts au Professeur H. Legrand, Paris, Cerf, p. 78-87.

(2006b), "Le synode des évêques: un débat inachevé ", dans G. Routhier et L. Villemin, dir., Nouveaux apprentissages pour l'Église. Mélanges offerts au Professeur H. Legrand, Paris, Cerf, p. 269294.

(2006c), "L'Assemblée extraordinaire de 1985 du synode des évêques. Moment charnière de relecture de Vatican II dans l'Église catholique ", dans P. Bordeyne et L. Villemin, dir., Vatican II et la théologie. Perspectives pour le XXI ${ }^{e}$ siècle, Paris, Cerf (Cogitatio fidei 254), p. 61-88.

(2010), "Un "mandatum docendi" dénié. Comment on interprète un silence», dans G. Routhier et G. Jobin, dir., L'Autorité et les autorités. L'herméneutique théologique de Vatican II, Paris, Cerf (Unam Sanctam, nouvelle série 3), p. 167-187.

(2019, à paraître), «La réforme de la curie romaine et la synodalité de l'Église », dans G. Routhier et J. FAmerée, Penser la réforme de l'Église, Paris, Cerf (Unam Sanctam).

Tinon, P. (2000), dir., Changer la papauté?, Paris, Cerf.

VRIEs, W. de (1974), Orient et Occident. Les structures ecclésiales vues dans l'histoire des sept premiers conciles oecuméniques, Paris, Cerf (Histoire des doctrines ecclésiologiques).

Winterkamp, K. (2003), Die Bischofkonferenz zwischen "affectiver" un effektiver Kollegialität, Münster.

\section{Résumé}

On parle abondamment de la «décentralisation» du gouvernement de l'Église catholique. Il y a une admission implicite, dans cette manière de parler, du fait que l'Église de Rome est le centre et que les autres Églises gravitent autour de ce centre. Et si l'on pensait autrement les choses, dans une perspective de communion entre les Églises. Cela conduirait probablement à envisager autrement la réforme des institutions «centrales » de gouvernement de l'Église universelle. Non seulement on revaloriserait les conférences épiscopales ou les assemblées régionales d'évêques, mais on envisagerait réellement une déconcentration des organismes de la curie. Telle est la proposition avancée dans cet article. 


\section{Abstract}

There is much talk on "decentralization" of the government of the Catholic Church. In this way of speaking, there is an implicit admission that the Church of Rome is the center and that other Churches revolve around this center. There is probably a different way to view the question, this time in a perspective of communion of churches all together in communion with the Church of Rome. This would probably lead to a different approach to the reform of the "central" institutions of the government of the universal Church, namely the roman curia. Not only would episcopal conferences or regional gatherings of bishops flourish with fresh vigour, but a real «deconcentrating" of the dicasteries of the roman dicasteries must be considered. This is the proposition put forward in this essay. 